\title{
Hepatobiliary Disorders in Celiac Disease: An Update
}

\author{
Kaushal K. Prasad, ${ }^{1,2}$ Uma Debi, ${ }^{3}$ Saroj K. Sinha, ${ }^{1}$ Chander K. Nain, ${ }^{1}$ and Kartar Singh ${ }^{1}$ \\ ${ }^{1}$ Department of Superspeciality of Gastroenterology, Postgraduate Institute of Medical Education and Research, \\ Chandigarh 160 012, India \\ ${ }^{2}$ Division of GE Histopathology, Department of Superspeciality of Gastroenterology \\ Postgraduate Institute of Medical Education and Research, Chandigarh 160 012, India \\ ${ }^{3}$ Department of Radiodiagnosis, Government Medical College \& Hospital, Sector 32, Chandigarh 160030, India
}

Correspondence should be addressed to Kaushal K. Prasad, kaushalkp10@hotmail.com

Received 6 July 2010; Revised 2 September 2010; Accepted 6 October 2010

Academic Editor: Takuji Torimura

Copyright ( $) 2011$ Kaushal K. Prasad et al. This is an open access article distributed under the Creative Commons Attribution License, which permits unrestricted use, distribution, and reproduction in any medium, provided the original work is properly cited.

This communication reviews recent literature and summarizes hepatobiliary abnormalities that may complicate the clinical course of celiac disease. A wide spectrum of hepatobiliary diseases has been described, including asymptomatic elevations of liver enzyme levels, nonspecific hepatitis, nonalcoholic fatty liver disease, and autoimmune and cholestatic liver disease. Moreover, in the majority of patients, liver enzyme levels will normalize on a gluten-free diet. In addition, celiac disease may be associated with rare hepatic complications, such as hepatic T-cell lymphoma. Because many celiac patients do not have overt gastrointestinal symptoms, a high index of suspicion is required. Simple methods of detecting celiac disease such as serum antibody tests help in the early identification of the disease, thus preventing serious complications of the disorder. The IgG DGP antibody test and IgA tTG antibody test used in combination are an excellent screening test for suspected cases of celiac disease.

\section{Introduction}

Celiac disease (CD), or gluten-sensitive enteropathy (GSE), is an autoimmune disorder characterized by reversible small bowel mucosal inflammation with villous atrophy affecting patients with a specific genetic predisposition (HLA DR3DQ2 and HLA DR4-DQ8). The mucosal lesion develops after ingestion of dietary gluten and recovers when glutencontaining cereals, wheat, rye, and barley, are withdrawn from the diet [1]. In untreated $\mathrm{CD}$, the characteristic abnormalities in the small bowel mucosa are villous atrophy, crypt hyperplasia, and an increased density of inflammatory cells in the epithelium and lamina propria [2-4]. The small bowel lesion of $\mathrm{CD}$ is a dynamic process whereby mucosal damage to the small intestine develops in three subsequent phases: (a) infiltrative phase, characterized solely by an increased number of intraepithelial lymphocytes; (b) hyperplastic phase, characterized by crypt hypertrophy; (c) destructive phase, which is characterized by progressive villous atrophy ultimately leading to the flattening of the mucosa [5].
Abnormal immune response to gliadin, genetic, and environmental factors plays a role in the pathogenesis of $\mathrm{CD}$. Infectious agents have been implicated in the pathogenesis of many autoimmune disorders. Transient infections or increased permeability of the mucosa may facilitate disease onset induced by the uptake of gluten peptides into a microenvironmental milieu in the small intestinal mucosa [6]. An environmental factor, such as an infectious agent, is thought to precipitate the disease via various pathogenic mechanisms, such as molecular mimicry, resulting in modulation of the host's immune tolerance [7]. Recently, two patients with onset of $\mathrm{CD}$ after resolution of acute hepatitis $B$ virus (HBV) infection have been reported in the literature [6]. Celiac disease has also been described in association with hepatitis C virus (HCV), rotavirus, adenovirus 12, and astrovirus as another immunologic manifestation of this infectious disease [8-11]. On the contrary, Plot et al. (2009) examined the association between serological evidence of past infection with Toxoplasma gondii, rubella virus, cytomegalovirus, Treponema pallidum, and EpsteinBarr virus and the coexistence of CD. The results implied 
that certain infections may generate an immunological environment that disfavours future appearance of certain autoimmune conditions such as CD [12]. Hence, understanding the relationship between infectious agents and autoimmune disorders is of importance that may assist in prediction, early diagnosis, and perhaps also the prevention of $\mathrm{CD}$ [7].

Population-based screening studies have shown that CD is very common and affects about one in 120 [13]. The diagnosis of $\mathrm{CD}$ is easily overlooked as patients can present with mild or atypical symptoms or the condition can even be clinically silent. Patients may present with only subtle, if any, symptoms [14], which is the main reason why the disease is highly underdiagnosed in India and elsewhere. In recent years, recognition of $\mathrm{CD}$ has been substantively improved with increasing awareness for CD amongst health care professionals and partly as a result of more modern serological assays for screening. The advent of serological methods for the detection of antibodies to gliadin, endomysial, and tissue transglutaminase has enabled large-scale screening for $\mathrm{CD}$, thus preventing serious complications of the disorder. The most common serological tests for the screening of $\mathrm{CD}$ are the indirect immunofluorescence (IFA) method of detecting endomysial antibodies (EmA) and ELISA methods of detecting antibodies to tissue transglutaminase (tTG) and gliadin $[15,16]$. Because of the limited sensitivity and specificity of gliadin antibody assays, use of this method is more limited than EmA and tTG. Reliance on EmA and tTG immunoassays, however, may allow cases of IgA-deficient CD to go undetected [17]. Both immunoassays may be of limited utility detecting IgG antibodies. IgG gliadin antibody tests are useful in establishing diagnosis of CD in IgA deficient patients. More recently, a new generation of promising assays detecting the presence of deamidated synthetic peptides of gliadin (DGP) has shown very high diagnostic performance equivalent to conventional tests $[18,19]$. The IgG DGP and IgA tTG in combination are an excellent screening algorithm for suspected cases of CD [19].

Although most symptomatic patients have symptoms related to the gastrointestinal tract, many extraintestinal manifestations have been described. The target organs include endocrine system [20-22], kidney [23, 24], skin [25], nervous system [26], and reproductive systems [27]. A number of hepatobiliary tract and pancreatic disorders have also been documented in patients with $\mathrm{CD}$ [28]. Some of these have been hypothesized to share common genetic factors or have a common immunopathogenesis, such as primary biliary cirrhosis ( $\mathrm{PBC})$, primary sclerosing cholangitis (PSC), and autoimmune forms of hepatitis or cholangitis [28]. Other hepatic changes in CD that may be associated with increased intestinal permeability could result in translocation of gut bacteria, kupffer cell stimulation, and production of tumor necrosis factor- $\alpha$ (TNF$\alpha$ ), proinflammatory cytokine, and reactive oxygen species, resulting in nonalcoholic steatohepatitis (NASH). It is also hypothesized that the malnutrition in $\mathrm{CD}$ might lead to chronic deficiency of a lipotropic factor (e.g., choline), with an associated pyridoxine deficiency, resulting in hepatic steatosis [29]. In addition, CD may be associated with rare hepatic complications, such as hepatic T-cell lymphoma [28]. Finally, pancreatic abnormalities may be caused by CD or coexist with $\mathrm{CD}$. Both endocrine and exocrine functions of the pancreas may be substantially changed in CD. We review the literature pertaining to hepatobiliary abnormalities that may be seen in association with celiac disease.

\section{Hepatobiliary Disorders}

Hepatic changes in patients with CD were initially described by Pollock in 1977 [30]. Since then the relationship between hepatobiliary dysfunction and CD is well established, ranging from asymptomatic or cryptogenic hypertransaminasemia to chronic liver disease (CLD) [31]. Thus, the hepatologist needs to consider $\mathrm{CD}$ in the differential of abnormal liver blood tests and to be aware of the clinical implications of this frequent disease in patients with liver disorders. The etiology is varying and multifactorial, such as genetic predisposition, precocity, and duration of exposure to gluten that may influence reversibility of liver damage [32].

\subsection{Asymptomatic Elevations of Liver Enzyme Levels.} Transaminases (ALT (alanine aminotransferase) and AST (aspartate aminotransferase)), $\gamma$-glutamyl transpeptidase $(\gamma$ GT), and alkaline phosphatase (ALP) are classically termed as liver enzymes; however, they can be found in almost every organ. Elevated levels of the transaminases ALT and AST are signs of disturbed permeability of the cells, in which these enzymes can be found. In contrast to ALT, which is mainly liver specific, the AST is found in other organs as well, for example, heart and skeletal muscle [33]. As such hypertransaminasemia has been observed in up to $40 \%$ of untreated celiac patients [34]. Celiac disease at diagnosis may present in $5 \%-10 \%$ of patients as an asymptomatic or cryptogenic hypertransaminasemia, as an expression of a mild liver dysfunction with a histological picture of nonspecific reactive hepatitis (celiac hepatitis) which frequently disappears after gluten suppression [32, 35-37]. At a mild elevation of liver enzymes, reevaluation is recommended; however, if an elevation persists and is suspicious for a liver disease, a specific workup is necessary [33].

2.2. Nonalcoholic Fatty Liver Disease. Nonalcoholic fatty liver disease (NAFLD) refers to a spectrum of diseases of the liver ranging from simple steatosis (i.e., fatty infiltration of the liver) to nonalcoholic steatohepatitis (i.e., steatosis with inflammation and hepatocyte necrosis (NASH)) to cirrhosis. Nonalcoholic fatty liver disease is the most common cause of elevated liver enzymes in adults and the most common cause of cryptogenic cirrhosis, which is cirrhosis that cannot be explained by hepatitis, alcohol abuse, toxin exposure, autoimmune disease, congenital liver disease, vascular outflow obstruction, or biliary tract disease [38].

Celiac disease has been reported in $4 \%-13 \%$ of cases with NASH [39-41]. The etiopathogenesis of hepatic steatosis in $\mathrm{CD}$ is uncertain. It has been suggested that malabsorption in 
CD might lead to chronic deficiency of a lipotropic factor (e.g., choline), with an associated pyridoxine deficiency, and hepatic steatosis might occur [29]. It is plausible that $\mathrm{CD}$, like small intestinal bacterial overgrowth (SIBO), can be associated with increased intestinal permeability [42]. The increased intestinal permeability could result in translocation of gut bacteria, kupffer cell stimulation, and production of tumor necrosis factor- $\alpha$ (TNF- $\alpha$ ), proinflammatory cytokine, and reactive oxygen species, resulting in NASH. Further studies are warranted to define the precise pathogenetic mechanism or mechanisms for NAFLD in CD.

2.3. Autoimmune Hepatobiliary Disorders. Autoimmune hepatobiliary disorders are frequently associated with $C D$, but they might remain undiagnosed because of lack of symptoms, absence of liver-specific autoantibodies, or misdiagnosis of celiac hepatitis. Acute hepatitis in celiac patients should induce one to suspect an autoimmune origin [43]. Patients with end-stage autoimmune liver disease, especially those who are HLA-DQ2 or HLA-DQ8 positive, had a high prevalence of $\mathrm{CD}$-associated antibodies [44]. Indeed, $\mathrm{CD}$ has been found in $3 \%-7 \%$ of patients with $\mathrm{PBC}$, in $3 \%-6 \%$ with autoimmune hepatitis (AIH), and in $2 \%$ $3 \%$ with PSC. Differently from cryptogenic liver injury, autoimmune liver dysfunction, found in $\mathrm{CD}$, does not usually improve after a gluten-free diet (GFD) [35]. Since $\mathrm{CD}$ is common among patients with autoimmune liver disease, screening autoimmune liver disease patients for CD is indicated. Although the magnitude of benefit from a GFD in reversing autoimmune liver disease in patients with $\mathrm{CD}$ is controversial, it may reduce the risk of further complications of CD [45].

2.3.1. Autoimmune Hepatitis and Cholangitis. Although AIH has been reported in only a limited number of case reports and population-based studies, the prevalence of $\mathrm{CD}$ has been found in 3\%-6\% with AIH [35, 45]. Conversely, patients with $\mathrm{CD}$ have a sixfold greater risk of AIH compared to the general population [46]. The two forms of AIH with different serological and clinical features can be found in association with CD. Indeed, the two well-defined forms of $\mathrm{AIH}$, found in CD, are AIH type 1, which is characterized by positivity for ANA/SMA and affects much more frequently adult patients, and AIH type 2, characterized by LKM and/or LC1 antibody positivity, usually confined to childhood CD $[47,48]$. Seronegative AIH has also been reported with CD [49]. In CD with AIH, GFD plus immunosuppressants determines a high remission rate. When clinical remission is reached, a prolonged immunosuppressive regimen induces a high sustained remission rate after treatment withdrawal [50].

Autoimmune cholangitis (antimitochondrial antibodynegative $\mathrm{PBC}$ ) is a rare chronic cholestatic liver disease. $\mathrm{CD}$ should be considered in all patients diagnosed with autoimmune cholangitis as a GFD may avoid the need for immunosuppressive therapy in affected patients [51].

2.3.2. Primary Sclerosing Cholangitis. An association between CD and PSC was first described in1988 by Hay et al. in a report of three patients with PSC later diagnosed with CD [52]. Thereafter, few other cases were reported $[53,54]$. It has been difficult to show good evidence for a response of the PSC to a gluten-free diet.

2.3.3. Primary Biliary Cirrhosis. The association between CD and $\mathrm{PBC}$ was first described in four patients by Logan et al. [55]. Numerous case reports followed formal epidemiological studies to determine the prevalence of $\mathrm{PBC}$ in patients with CD [56]. Although an association between PBC and $\mathrm{CD}$ has been reported, there are still conflicting data concerning this issue [57]. Considering the malabsorption, weight loss and osteoporosis are clinical manifestations common to both entities, screening for $\mathrm{CD}$ in $\mathrm{PBC}$ and vice versa, for antimitochondrial antibodies are advisable in adult $\mathrm{CD}$ patients for early diagnosis and institution of specific therapeutic measures [57].

2.4. Miscellaneous Hepatobiliary Disorders in Celiac Disease. Celiac disease can present great clinical heterogeneity. Its association with a series of intestinal and nonintestinal diseases, whether immunologically mediated or otherwise, presents a higher than normal frequency. Celiac disease has increasingly been reported with a variety of other liver diseases.

2.4.1. Hemochromatosis. Celiac disease and hereditary hemochromatosis $(\mathrm{HH})$ are genetic disorders with opposite effects on iron absorption and probably on the absorption of some other divalent metals including copper [58]. Hereditary hemochromatosis is the most common autosomal recessive disease in the Caucasian population and is characterised by an iron overload state. Celiac disease, or GSE, on the other hand is frequently associated with iron deficiency anemia. The concomitant presence of $\mathrm{HH}$ and $\mathrm{CD}$ is rare, and susceptibility to both is associated with genes in the major histocompatibility complex (MHC) on the short arm of chromosome $6[59,60]$. However, their MHC association is quite distinct. CD is associated with the HLA-DQ2 allele as well as non-HLA genes, whereas $\mathrm{HH}$ is associated with the HLA-A3 and B7 alleles of the MHC region [61]. It is not clear whether there is a genetic relationship between the two conditions or their association merely coincidence based on their higher frequencies in the general population. i?ehlt?;

Occult CD may compensate for increased divalentmetal transporter 1 (DMT1) expression in a specific subset of individuals with homozygous $\mathrm{C} 282 \mathrm{Y}$ mutations in the hemochromatosis (HFE) gene, thus contributing to the low penetrance of $\mathrm{HH}$ [62]. On the other hand, HFE mutations do not constitute a protective factor against the development of iron deficiency, which seems to be mainly determined by the severity of the intestinal lesions [63].

2.4.2. Gallstone Diseases. Impaired gallbladder motility has been reported also in patients with $\mathrm{CD}$ in relation to reduced secretion of enteric hormones and/or decreased gallbladder sensitivity to them. In particular, untreated celiacs showed low postprandial cholecystokinin and increased 
fasting somatostatin levels [64]. In spite of these physiological alterations, there does not appear to be a significant predisposition to gallstones in CD except for elderly celiac [65].

2.4.3. Hepatic Vein Obstruction. The association of BuddChiari syndrome (BCS) and CD is rare and defined by obstruction of the hepatic vein. All but one of the reported cases had thrombosis of hepatic veins, and one patient had membranous obstruction of inferior vena cava (IVC) [6668]. Celiac disease should be considered especially in the event of BCS and malabsorption. The long-term outcome is favorable with a GFD and antivitamin K treatment [69]. A percutaneous balloon angioplasty was performed, with satisfactory outcome in case of membranous obstruction of the inferior vena cava [68].

2.4.4. Noncirrhotic Portal Hypertension. Nodular regenerative hyperplasia (NRH) of the liver is a rare disorder that is characterized by a nodular transformation of the hepatic parenchyma without fibrous septa between the nodules, is often associated with connective tissue disorders, hematological malignancy, or drugs, and is a cause of noncirrhotic portal hypertension $[70,71]$. Antiphospholipid antibodies (aPL) are a heterogeneous group of circulating autoantibodies including anticardiolipins found in the sera of healthy subjects and patients with autoimmune and infectious diseases. IgA anticardiolipin antibodies were reported recently in celiac patients with $\mathrm{NRH}[72,73]$.

Idiopathic portal hypertension (IPH) or noncirrhotic portal fibrosis (NCPF) is a disorder generally classified as a noncirrhotic portal hypertension of unknown etiology and is clinically characterized by portal hypertension, splenomegaly, and pancytopenia $[74,75]$. Idiopathic portal hypertension is a heterogeneous and multifactorial disorder with a potential genetic contribution, seen most often in the Indian subcontinent and in East Asia [76]. The association of CD with IPH has been recently reported in the literature $[77,78]$. The improvement of portal hypertension with a GFD implicates a causal relationship between portal hypertension and increased inflammatory reactions in CD [79]. The coexistence of CD and IPH suggests that there may be an immunological link between these two conditions [78]. Therefore, the testing for CD in patients with IPH is warranted.

2.4.5. Hepatic Malignancies. Celiac disease is associated with intestinal lymphoma and other forms of cancer, especially adenocarcinoma of the small intestine, of the pharynx, and of the esophagus. Enteropathy-associated T-cell lymphoma (EATL) is a rare form of high-grade, T-cell non-Hodgkin lymphoma (NHL) of the upper small intestine that is specifically associated with CD. Enteropathy-associated T-cell lymphoma derives from a clonal proliferation of intraepithelial lymphocytes (IELs) and is often disseminated at diagnosis. Extraintestinal presentations are not uncommon in the liver, spleen, thyroid, skin, nasal sinus, and brain [80]. Lymphoma in the liver is apparently secondary to intestinal lymphoma, complicating celiac disease [30]. In general, involvement of the liver in CD patients with lymphoma is limited and overshadowed by the clinical course of the intestinal disease. The outlook of EATL is poor. Hepatocellular carcinoma (HCC) in one patient and hepatosplenic lymphoma, a rare type of peripheral T-cell lymphoma with rearrangement of the $\gamma \delta$ T-cell receptor, have been reported [30,81,82].

2.4.6. End-Stage Liver Disease. Although rare, severe hepatic damage or failure can develop in association with CD [31]. In a few cases, when CD is diagnosed, a more severe liver injury, characterized by a cryptogenic chronic hepatitis or liver cirrhosis, was present [35]. Celiac disease is up to 10 times more frequent among patients with CLD [83]. Severe liver failure and decompensated cryptogenic liver cirrhosis are potentially treatable with institution of a GFD. Dietary treatment may prevent progression to hepatic failure, even in cases in which liver transplantation is considered [84]. Therefore, all patients with cryptogenic cirrhosis should be screened for CD-related antibodies before proceeding to a liver transplant.

Increased intestinal permeability, SIBO, kupffer cell abnormalities, direct toxicity of gliadin peptides, or a gliadinstimulated immunopathological process may have a role to play in the progression of CLD among celiac patients $[83,84]$. The patients with CLD have a higher likelihood of false positive results regarding anti-tTG antibodies, which is due, in some instances, to increased immunoglobulins concentrations and, in most individuals, to the role of transglutaminase in liver fibrosis mechanisms [85]. The specificity and sensitivity of tTG assays have been questioned, and several publications describe positive tTG antibody results in autoimmune diseases other than CD, while the samples are negative for EmA [86-88]. Furthermore, the finding of false positive results for anti-tTG antibodies is a relevant problem for antibodies to guinea pig tTG, whereas the number of false positives is far much lower for antibodies to recombinant human tTG, the usually routine test for CD screening. More recently, a new generation of promising assays detecting the presence of deamidated synthetic peptides of gliadin (DGP) has shown very high diagnostic performance equivalent to conventional tests $[18,19]$. The combination of two assays makes diagnostic accuracy higher. Therefore, a serologic screening by combining tTG IgA with DGP IgG or EmA testing could be considered as the best initial test for CD [19].

\section{Conclusions}

In conclusion, a number of hepatobiliary disorders have been documented in patients with celiac disease. Consequently, newly onset celiac disease should be rigorously checked for asymptomatic hepatic damage and conversely, any cryptogenic liver disorder, including end-stage liver disease, should be investigated for celiac disease. Presently, it is difficult to establish if the two main kinds of hepatic injury (cryptogenic and autoimmune) found in celiac disease are discrete entities with a different pathogenesis or if they are an expression of the same disorder, where genetic factors and the duration of gluten exposure may determine the severity and the pattern 
of hepatic injury. Several communications of isolated cases of rare hepatobiliary diseases, which probably, only reflect a fortuitous association with celiac disease, have been cited in the literature.

\section{References}

[1] J. S. Trier, "Medical progress: celiac sprue," New England Journal of Medicine, vol. 325, no. 24, pp. 1709-1719, 1991.

[2] K. K. Prasad, B. R. Thapa, C. K. Nain, A. K. Sharma, and K. Singh, "Brush border enzyme activities in relation to histological lesion in pediatric celiac disease," Journal of Gastroenterology and Hepatology, vol. 23, no. 8, pp. e348-e352, 2008.

[3] K. K. Prasad, B. R. Thapa, C. K. Nain, and K. Singh, "Assessment of the diagnostic value of duodenal bulb histology in patients with celiac disease, using multiple biopsy sites," Journal of Clinical Gastroenterology, vol. 43, no. 4, pp. 307-311, 2009.

[4] K. K. Prasad, B. R. Thapa, C. K. Nain, and K. Singh, "Assessment of histologic lesion variability of the duodenal mucosa in children with celiac disease," World Journal of Pediatrics, vol. 6, pp. 60-64, 2010.

[5] M. N. Marsh, "Grains of truth: evolutionary changes in small intestinal mucosa in response to environmental antigen challenge," Gut, vol. 31, no. 1, pp. 111-114, 1990.

[6] S. Soto Iglesias, S. Vázquez Rodríguez, J. L. Ulla Rocha et al., "Onset of celiac disease after acute hepatitis B infection," Gastroenterologia y Hepatologia, vol. 33, no. 1, pp. 17-20, 2010.

[7] L. Plot and H. Amital, "Infectious associations of Celiac disease," Autoimmunity Reviews, vol. 8, no. 4, pp. 316-319, 2009.

[8] I. Aguancha, J. M. Valera, C. Hurtado, G. Smok, and J. Brahm, "Chronic hepatitis $\mathrm{C}$ and celiac sprue: an infrequent association," Gastroenterologia y Hepatologia, vol. 27, no. 7, pp. 408-410, 2004.

[9] L. C. Stene, M. C. Honeyman, E. J. Hoffenberg et al., "Rotavirus infection frequency and risk of celiac disease autoimmunity in early childhood: a longitudinal study," American Journal of Gastroenterology, vol. 101, no. 10, pp. 2333-2340, 2006.

[10] M. Lawler, P. Humphries, C. O’Farrelly et al., "Adenovirus 12 E1A gene detection by polymerase chain reaction in both the normal and coeliac duodenum," Gut, vol. 35, no. 9, pp. 12261232, 1994.

[11] S. L. Smits, M. Van Leeuwen, A. A. Van Der Eijk et al., "Human astrovirus infection in a patient with new-onset celiac disease," Journal of Clinical Microbiology, vol. 48, no. 9, pp. 3416-3418, 2010.

[12] L. Plot, H. Amital, O. Barzilai, M. Ram, B. Nicola, and Y. Shoenfeld, "Infections may have a protective role in the etiopathogenesis of celiac disease," Annals of the New York Academy of Sciences, vol. 1173, pp. 670-674, 2009.

[13] S. Lal, C. K. Nain, K. K. Prasad, and B. R. Thapa, "Celiac disease in Chandigarh: a population survey," Indian Journal of Gastroenterology, vol. 24, supplement 1, p. A39, 2005.

[14] G. R. Corazza, M. Frisoni, E. A. Treggiari et al., "Subclinical celiac sprue: increasing occurrence and clues to its diagnosis," Journal of Clinical Gastroenterology, vol. 16, no. 1, pp. 16-21, 1993.

[15] T. P. Chorzelski, E. H. Beutner, and J. Sulej, "IgA antiendomysium antibody. A new immunological marker of dermatitis herpetiformis and coeliac disease," British Journal of Dermatology, vol. 111, no. 4, pp. 395-402, 1984.

[16] D. Schuppan and E. G. Hahn, "IgA anti-tissue transglutaminase: setting the stage for coeliac disease screening," European Journal of Gastroenterology and Hepatology, vol. 13, no. 6, pp. 635-637, 2001.

[17] A. Pacht, N. Sinai, L. Hornstein, V. Kumar, N. Ish-Shalom, and A. Lerner, "The diagnostic reliability of anti-endomysial antibody in celiac disease: the north Israel experience," Israel Journal of Medical Sciences, vol. 31, no. 4, pp. 218-220, 1995.

[18] S. Niveloni, E. Sugai, A. Cabanne et al., "Antibodies against synthetic deamidated gliadin peptides as predictors of celiac disease: prospective assessment in an adult population with a high pretest probability of disease," Clinical Chemistry, vol. 53, no. 12, pp. 2186-2192, 2007.

[19] E. Sugai, M. L. Moreno, H. J. Hwang et al., "Celiac disease serology in patients with different pretest probabilities: is biopsy avoidable?" World Journal of Gastroenterology, vol. 16, no. 25, pp. 3144-3152, 2010.

[20] P. Collin, K. Kaukinen, M. Välimäki, and J. Salmi, "Endocrinological disorders and celiac disease," Endocrine Reviews, vol. 23, no. 4, pp. 464-483, 2002.

[21] E. Ferrante, C. Giavoli, L. Elli et al., "Evaluation of GH-IGFI axis in adult patients with coeliac disease," Hormone and Metabolic Research, vol. 42, no. 1, pp. 45-49, 2010.

[22] M. Delvecchio, A. De Bellis, R. Francavilla et al., "Antipituitary antibodies in children with newly diagnosed celiac disease: a novel finding contributing to linear-growth impairment," American Journal of Gastroenterology, vol. 105, no. 3, pp. 691-696, 2010.

[23] K. D. Jhaveri, V. D. D’Agati, R. Pursell, and D. Serur, "Coeliac sprue-associated membranoproliferative glomerulonephritis (MPGN)," Nephrology Dialysis Transplantation, vol. 24, no. 11, pp. 3545-3548, 2009.

[24] H. K. Smerud, B. Fellström, R. Hällgren, S. Osagie, P. Venge, and G. Kristjánsson, "Gluten sensitivity in patients with IgA nephropathy," Nephrology Dialysis Transplantation, vol. 24, no. 8, pp. 2476-2481, 2009.

[25] F. Zingone, C. Bucci, R. Tortora et al., "Body mass index and prevalence of skin diseases in adults with untreated coeliac disease," Digestion, vol. 80, no. 1, pp. 18-24, 2009.

[26] H. J. Freeman, "Neurological disorders in adult celiac disease," Canadian Journal of Gastroenterology, vol. 22, no. 11, pp. 909$911,2008$.

[27] K. K. Prasad, S. Siwatch, S. K. Sinha, C. K. Nain, and K. Singh, "Celiac disease and reproductive disorders: association is often neglected," Gastroenterology Today, vol. 11, pp. 50-54, 2007.

[28] H. J. Freeman, "Hepatobiliary and pancreatic disorders in celiac disease," World Journal of Gastroenterology, vol. 12, no. 10, pp. 1503-1508, 2006.

[29] J. E. Naschitz, D. Yeshurun, E. Zuckerman, E. Arad, and J. H. Boss, "Massive hepatic steatosis complicating adult celiac disease: report of a case and review of the literature," American Journal of Gastroenterology, vol. 82, no. 11, pp. 1186-1189, 1987.

[30] D. J. Pollock, "The liver in coeliac disease," Histopathology, vol. 1, no. 6, pp. 421-430, 1977.

[31] T. H. Casswall, N. Papadogiannakis, S. Ghazi, and A. Németh, "Severe liver damage associated with celiac disease: findings in six toddler-aged girls," European Journal of Gastroenterology and Hepatology, vol. 21, no. 4, pp. 452-459, 2009.

[32] M. D. Cantarero Vallejo, J. Gómez Camarero, L. Menchén, J. A. Pajares Díaz, and O. Lo Iacono, "Liver damage and celiac 
disease," Revista Espanola de Enfermedades Digestivas, vol. 99, no. 11, pp. 648-652, 2007.

[33] P. M. Lepper and J.-F. Dufour, "Elevated transaminases-what to do if everything was done?" Praxis, vol. 98, no. 6, pp. 330334, 2009.

[34] A. B. Villares, J. A. M. Monteagudo, R. M. Borque, and R. M. Otero, "Hepatic involvement in celiac disease," Gastroenterologia y Hepatologia, vol. 31, no. 1, pp. 25-28, 2008.

[35] U. Volta, "Pathogenesis and clinical significance of liver injury in celiac disease," Clinical Reviews in Allergy and Immunology, vol. 36, no. 1, pp. 62-70, 2009.

[36] A. Rubio-Tapia and J. A. Murray, "The liver in celiac disease," Hepatology, vol. 46, no. 5, pp. 1650-1658, 2007.

[37] G. Maggiore and S. Caprai, "Liver involvement in celiac disease," Indian Journal of Pediatrics, vol. 73, no. 9, pp. 809811,2006

[38] M. Bayard, J. Holt, and E. Boroughs, "Nonalcoholic fatty liver disease," American Family Physician, vol. 73, no. 11, pp. 19611969, 2006.

[39] J. M. Valera, C. Hurtado, J. Poniachik, P. Abumohor, and J. Brahm, "Study of celiac disease in patients with non-alcoholic fatty liver and autoimmune hepatic diseases," Gastroenterologia y Hepatologia, vol. 31, no. 1, pp. 8-11, 2008.

[40] M. T. Bardella, L. Valenti, C. Pagliari et al., "Searching for coeliac disease in patients with non-alcoholic fatty liver disease," Digestive and Liver Disease, vol. 36, no. 5, pp. 333336, 2004.

[41] A. Franzese, M. P. Iannucci, G. Valerio et al., "Atypical celiac disease presenting as obesity-related liver dysfunction," Journal of Pediatric Gastroenterology and Nutrition, vol. 33, no. 3, pp. 329-332, 2001.

[42] L. Miele, V. Valenza, G. La Torre et al., "Increased intestinal permeability and tight junction alterations in nonalcoholic fatty liver disease," Hepatology, vol. 49, no. 6, pp. 1877-1887, 2009.

[43] S. Caprai, P. Vajro, A. Ventura, M. Sciveres, and G. Maggiore, "Autoimmune liver disease associated with celiac disease in childhood: a multicenter study," Clinical Gastroenterology and Hepatology, vol. 6, no. 7, pp. 803-806, 2008.

[44] A. Rubio-Tapia, A. S. Abdulkarim, R. H. Wiesner, S. B. Moore, P. K. Krause, and J. A. Murray, "Celiac disease autoantibodies in severe autoimmune liver disease and the effect of liver transplantation," Liver International, vol. 28, no. 4, pp. 467476, 2008 .

[45] F. Mirzaagha, S. H. Azali, F. Islami et al., "Coeliac disease in autoimmune liver disease: a cross-sectional study and a systematic review," Digestive and Liver Disease, vol. 42, no. 9, pp. 620-623, 2010.

[46] J. F. Ludvigsson, P. Elfström, U. BroomÉ, A. Ekbom, and S. M. Montgomery, "Celiac disease and risk of liver disease: a general population-based study," Clinical Gastroenterology and Hepatology, vol. 5, no. 1, pp. 63-69.e1, 2007.

[47] D. Villalta, D. Girolami, E. Bidoli et al., "High prevalence of celiac disease in autoimmune hepatitis detected by antitissue tranglutaminase autoantibodies," Journal of Clinical Laboratory Analysis, vol. 19, no. 1, pp. 6-10, 2005.

[48] U. Volta, L. De Franceschi, N. Molinaro et al., "Frequency and significance of anti-gliadin and anti-endomysial antibodies in autoimmune hepatitis," Digestive Diseases and Sciences, vol. 43, no. 10, pp. 2190-2195, 1998.

[49] M. A. Quail, R. K. Russell, C. Bellamy, G. Mieli-Vergani, and P. M. Gillett, "Seronegative autoimmune hepatitis presenting after diagnosis of coeliac disease: a case report," European
Journal of Gastroenterology and Hepatology, vol. 21, no. 5, pp. 576-579, 2009.

[50] A. R. Di Biase, A. Colecchia, E. Scaioli et al., "Autoimmune liver diseases in a paediatric population with coeliac diseasea 10-year single-centre experience," Alimentary Pharmacology and Therapeutics, vol. 31, no. 2, pp. 253-260, 2010.

[51] R. E. Sedlack, T. C. Smyrk, A. J. Czaja, and J. A. Talwalkar, "Celiac disease-associated autoimmune cholangitis," American Journal of Gastroenterology, vol. 97, no. 12, pp. 3196-3198, 2002.

[52] J. E. Hay, R. H. Wiesner, R. G. Shorter, N. F. LaRusso, and W. P. Baldus, "Primary sclerosing cholangitis and celiac disease. A novel association," Annals of Internal Medicine, vol. 109, no. 9, pp. 713-717, 1988.

[53] U. Volta, L. Rodrigo, A. Granito et al., "Celiac disease in autoimmune cholestatic liver disorders," American Journal of Gastroenterology, vol. 97, no. 10, pp. 2609-2613, 2002.

[54] I. Venturini, R. Cosenza, L. Miglioli et al., "Adult celiac disease and primary sclerosing cholangitis: two case reports," HepatoGastroenterology, vol. 45, no. 24, pp. 2344-2347, 1998.

[55] R. F. A. Logan, A. Ferguson, N. D. C. Finlayson, and D. G. Weir, "Primary biliary cirrhosis and coeliac disease. An association?" Lancet, vol. 1, no. 8058, pp. 230-233, 1978.

[56] W. Dickey, S. A. McMillan, and M. E. Callender, "High prevalence of celiac spree among patients with primary biliary cirrhosis," Journal of Clinical Gastroenterology, vol. 25, no. 1, pp. 328-329, 1997.

[57] A. Floreani, C. Betterle, A. Baragiotta et al., "Prevalence of coeliac disease in primary biliary cirrhosis and of antimitochondrial antibodies in adult coeliac disease patients in Italy," Digestive and Liver Disease, vol. 34, no. 4, pp. 258-261, 2002.

[58] C. Whittington, "The C282Y mutation may have been positively selected as it mitigates the infertility of celiac disease," Medical Hypotheses, vol. 66, no. 4, pp. 769-772, 2006.

[59] S. Bevan, S. Popat, C. P. Braegger et al., "Contribution of the MHC region to the familial risk of coeliac disease," Journal of Medical Genetics, vol. 36, no. 9, pp. 687-690, 1999.

[60] C. Q. Edwards, M. M. Dadone, M. H. Skolnick, and J. P. Kushner, "Hereditary hemochromatosis," Clinical Hematology, vol. 11, pp. 411-435, 1982.

[61] J. R. Butterworth, B. T. Cooper, W. M. C. Rosenberg et al., "The role of hemochromatosis susceptibility gene mutations in protecting against iron deficiency in celiac disease," Gastroenterology, vol. 123, no. 2, pp. 444-449, 2002.

[62] A. Geier, C. Gartung, I. Theurl et al., "Occult celiac disease prevents penetrance of hemochromatosis," World Journal of Gastroenterology, vol. 11, no. 21, pp. 3323-3326, 2005.

[63] D. Barisani, S. Ceroni, S. Del Bianco, R. Meneveri, and M. T. Bardella, "Hemochromatosis gene mutations and iron metabolism in celiac disease," Haematologica, vol. 89, no. 11, pp. 1299-1305, 2004.

[64] M. Fraquelli, M. Pagliarulo, A. Colucci, S. Paggi, and D. Conte, "Gallbladder motility in obesity, diabetes mellitus and coeliac disease," Digestive and Liver Disease, vol. 35, no. 3, pp. S12S16, 2003.

[65] H. Freeman, M. Lemoyne, and P. Pare, "Coeliac disease," Best Practice and Research in Clinical Gastroenterology, vol. 16, no. 1, pp. 37-49, 2002.

[66] F. Ben Hriz, H. Habbassi, N. Maamouri et al., "Budd-Chiari syndrome associated with celiac disease," Revue de Medecine Interne, vol. 31, no. 2, pp. 160-162, 2010.

[67] R. Kochhar, I. Masoodi, U. Dutta et al., "Celiac disease and Budd Chiari syndrome: report of a case with review of 
literature," European Journal of Gastroenterology and Hepatology, vol. 21, no. 9, pp. 1092-1094, 2009.

[68] F. Martínez, M. Berenguer, M. Prieto, H. Montes, M. Rayón, and J. Berenguer, "Budd-Chiari syndrome caused by membranous obstruction of the inferior vena cava associated with coeliac disease," Digestive and Liver Disease, vol. 36, no. 2, pp. 157-162, 2004.

[69] E. Gelsi, F. Ruitord, M.-C. Saint-Paul, J. Filippi, K. Arab, and X. Hébuterne, "Association of Budd-Chiari syndrome with a coeliac disease in patient native from North Africa," Gastroenterologie Clinique et Biologique, vol. 28, no. 10, pp. 903-905, 2004.

[70] E. Biecker, J. Trebicka, H.-P. Fischer, T. Sauerbruch, and F. Lammert, "Portal hypertension and nodular regenerative hyperplasia in a patient with celiac disease," Zeitschrift fur Gastroenterologie, vol. 44, no. 5, pp. 395-398, 2006.

[71] F. Tinsa, I. Brini, A. El May, D. Bousnina, K. Boussetta, and S. Bousnina, "Nodular regenerative hyperplasia of the liver, coeliac disease and Sjogren's syndrome in a child," Gastroenterologie Clinique et Biologique, vol. 34, no. 1, pp. 4042, 2010.

[72] E. L. R. Cancado, D. M. Medeiros, M. M. Deguti et al., "Celiac disease associated with nodular regenerative hyperplasia, pulmonary abnormalities, and IgA anticardiolipin antibodies," Journal of Clinical Gastroenterology, vol. 40, no. 2, pp. 135-139, 2006.

[73] A. Austin, E. Campbell, P. Lane, and E. Elias, "Nodular regenerative hyperplasia of the liver and coeliac disease: potential role of IgA anticardiolipin antibody," Gut, vol. 53, no. 7, pp. 1032-1034, 2004.

[74] M. Okudaira, M. Ohbu, and K. Okuda, "Idiopathic portal hypertension and its pathology," Seminars in Liver Disease, vol. 22, no. 1, pp. 59-71, 2002.

[75] K. K. Prasad, "Histopathological changes in liver diseases," in Liver: A Complete Book on Hepato-Pancreato-Biliary Diseases, M. A. Mahtab and S. Rahman, Eds., pp. 69-106, Elsevier, Chennai, India, 2009.

[76] R. K. Dhiman, Y. Chawla, R. K. Vasishta et al., "Non-cirrhotic portal fibrosis (idiopathic portal hypertension): experience with 151 patients and a review of the literature," Journal of Gastroenterology and Hepatology, vol. 17, no. 1, pp. 6-16, 2002.

[77] B. C. Sharma, D. K. Bhasin, and R. Nada, "Association of celiac disease with non-cirrhotic portal fibrosis," Journal of Gastroenterology and Hepatology, vol. 21, no. 1, pp. 332-334, 2006.

[78] F. Zamani, A. Amiri, R. Shakeri, A. Zare, and M. Mohamadnejad, "Celiac disease as a potential cause of idiopathic portal hypertension: a case report," Journal of Medical Case Reports, vol. 3, article no. 68, 2009.

[79] B. Kara and M. Sandikci, "Successful treatment of portal hypertension and hypoparathyroidism with a gluten-free diet," Journal of Clinical Gastroenterology, vol. 41, no. 7, pp. 724-725, 2007.

[80] C. Catassi, I. Bearzi, and G. K. T. Holmes, "Association of celiac disease and intestinal lymphomas and other cancers," Gastroenterology, vol. 128, no. 4, pp. S79-S86, 2005.

[81] N. L. Harris, E. S. Jaffe, H. Stein et al., "A revised EuropeanAmerican classification of lymphoid neoplasms: a proposal from the International Lymphoma Study Group," Blood, vol. 84, no. 5, pp. 1361-1392, 1994.

[82] J.-P. Farcet, P. Gaulard, J.-P. Marolleau et al., "Hepatosplenic T-cell lymphoma: sinusal/sinusoidal localization of malignant cells expressing the T-cell receptor $\gamma \delta$," Blood, vol. 75, no. 11, pp. 2213-2219, 1990.
[83] F. M. Stevens and R. M. McLoughlin, "Is coeliac disease a potentially treatable cause of liver failure?” European Journal of Gastroenterology and Hepatology, vol. 17, no. 10, pp. 10151017, 2005.

[84] K. Kaukinen, L. Halme, P. Collin et al., "Celiac disease in patients with severe liver disease: gluten-free diet may reverse hepatic failure," Gastroenterology, vol. 122, no. 4, pp. 881-888, 2002.

[85] D. Villalta, M. Crovatto, S. Stella, E. Tonutti, R. Tozzoli, and N. Bizzaro, "False positive reactions for IgA and IgG anti-tissue transglutaminase antibodies in liver cirrhosis are common and method-dependent," Clinica Chimica Acta, vol. 356, no. 1-2, pp. 102-109, 2005.

[86] M. Sárdy, M. Csikós, C. Geisen et al., "Tissue transglutaminase ELISA positivity in autoimmune disease independent of gluten-sensitive disease," Clinica Chimica Acta, vol. 376, no. 12, pp. 126-135, 2007.

[87] N. Bizzaro, M. Tampoia, D. Villalta et al., "Low specificity of anti-tissue transglutaminase antibodies in patients with primary biliary cirrhosis," Journal of Clinical Laboratory Analysis, vol. 20, no. 5, pp. 184-189, 2006.

[88] L. M. Luft, S. G. Barr, L. O. Martin, E. K. L. Chan, and M. J. Fritzler, "Autoantibodies to tissue transglutaminase in Sjögren's syndrome and related rheumatic diseases," Journal of Rheumatology, vol. 30, no. 12, pp. 2613-2619, 2003. 


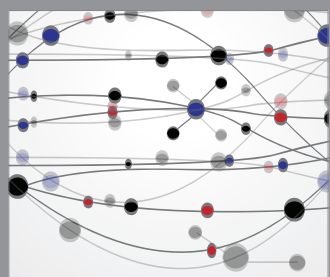

The Scientific World Journal
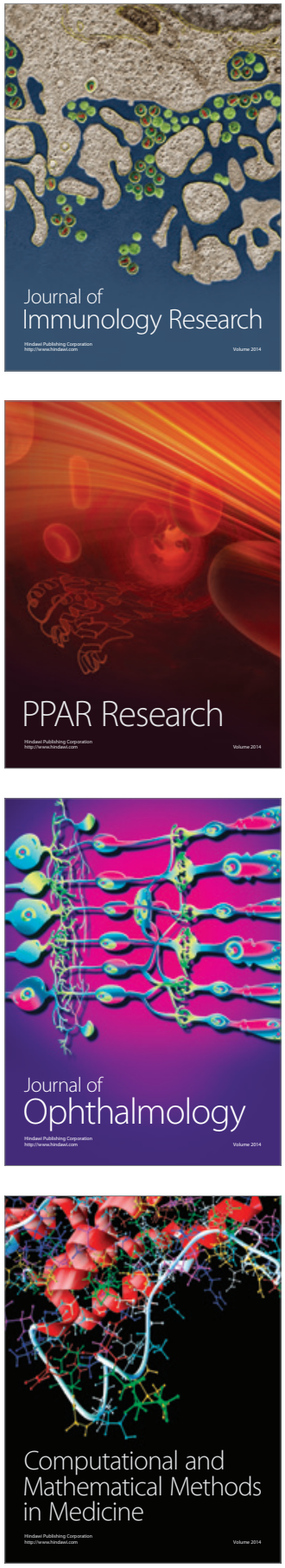

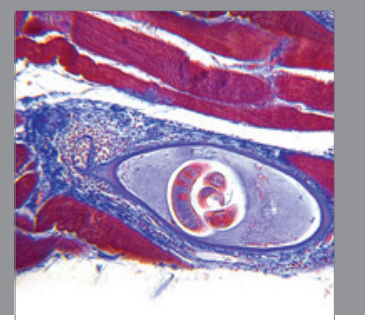

Gastroenterology

Research and Practice
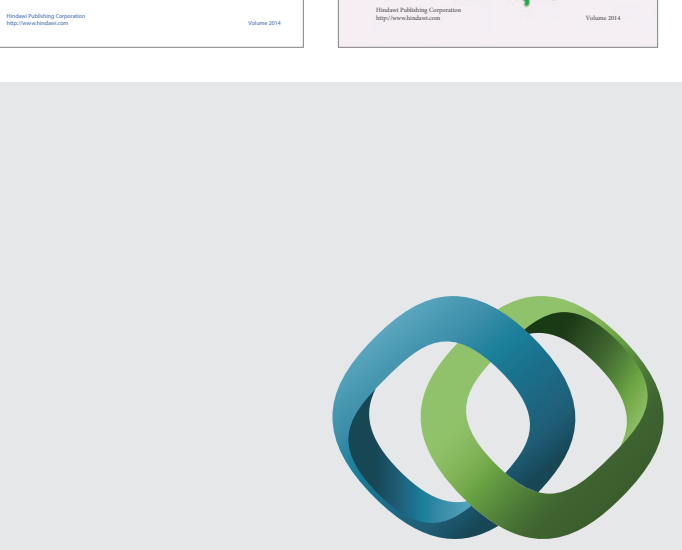

\section{Hindawi}

Submit your manuscripts at

http://www.hindawi.com
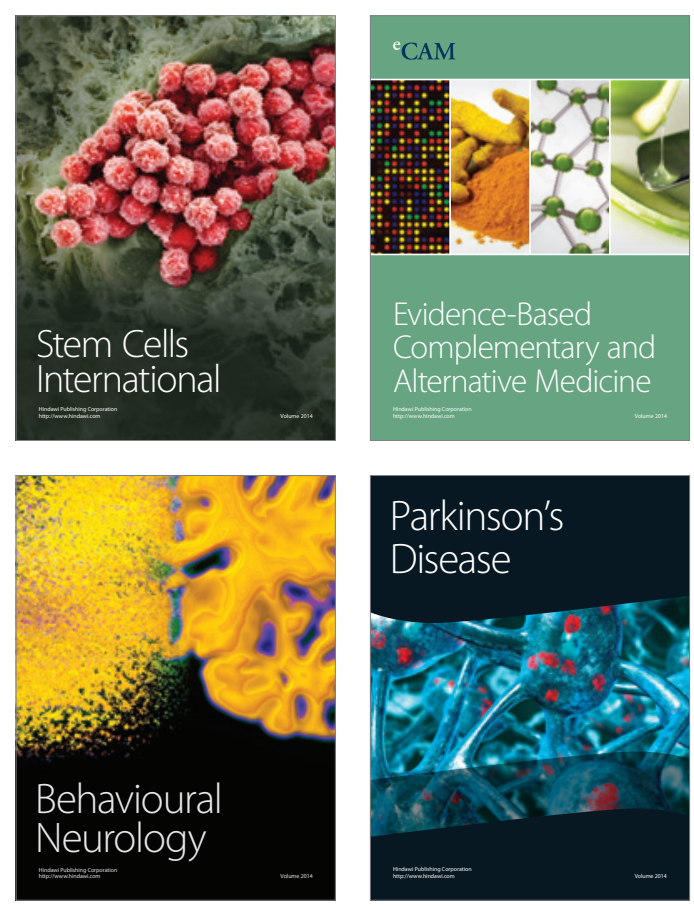

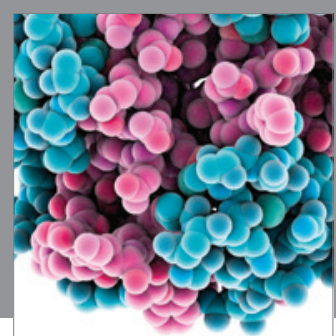

Journal of
Diabetes Research

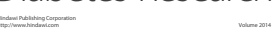

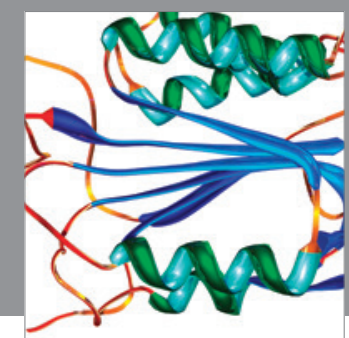

Disease Markers
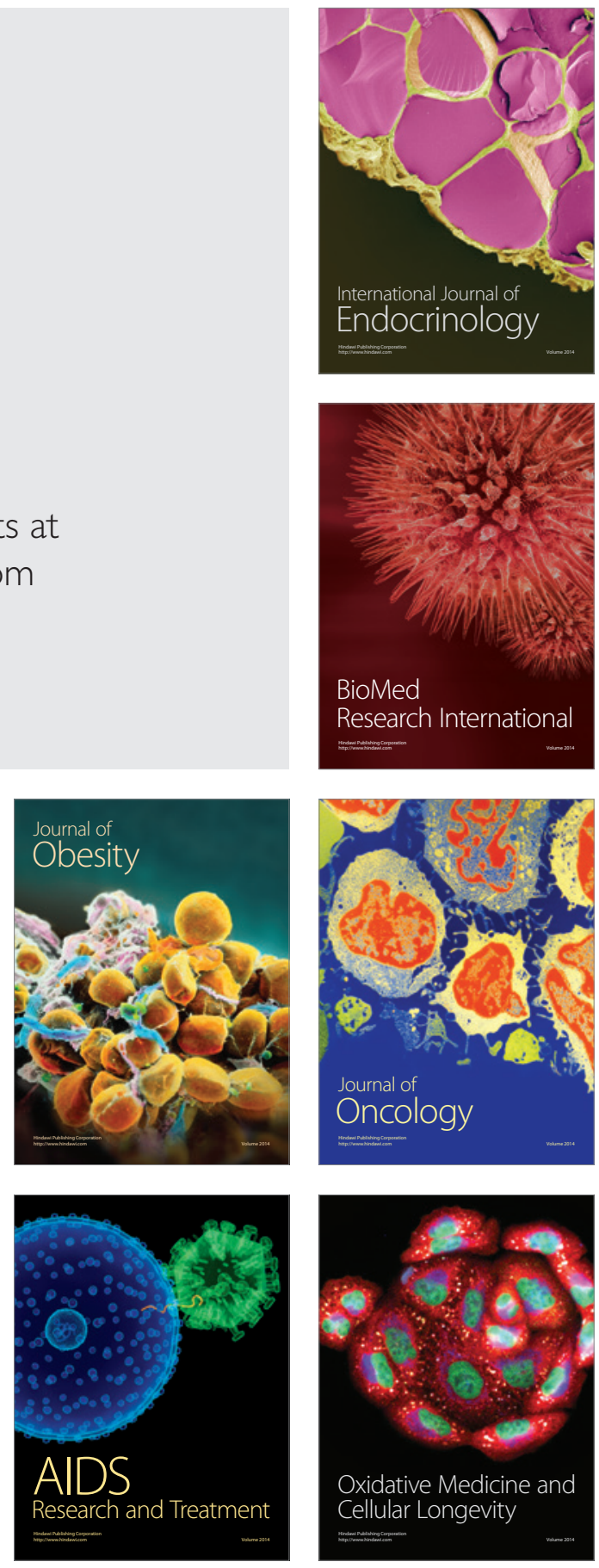